\title{
Oversensing in a Single Chamber Implantable Cardioverter Defibrillator
}

\author{
Paul A. Levine, $\mathrm{MD}^{1^{*}}$, Tracy Finegan, $\mathrm{NP}^{2}$, Jason C. Bradfield, MD ${ }^{3}$, Robin Y. Wachsner, MD \\ 1 Device Clinic, Department of Cardiology, Olive View UCLA Medical Center, Sylmar, California, USA \\ ${ }^{2}$ Heart Failure Clinic, Department of Cardiology, Olive View UCLA Medical Center, Sylmar, California, USA \\ ${ }^{3}$ EP Lab, Department of Cardiology, Olive View UCLA Medical Center, Sylmar, California, USA \\ ${ }^{4}$ Department of Cardiology, Cardiology Olive View UCLA Medical Center, Sylmar, California, USA
}

\begin{abstract}
A patient is seen in routine follow up for his ICD implanted for primary prevention. He is clinically stable and has not experienced any therapy. Upon routine interrogation, the system reports multiple episodes of oversensing. Is this a concern and if so, what can be done about it?
\end{abstract}

Copyright @ 2015 Science International Corp.

\section{Key Words}

Oversensing - Far Field P waves - Implantable cardioverter defibrillator

\section{Introduction}

A 57-year-old patient with a history of ischemic cardiomyopathy with a left ventricular ejection fraction of $25-30 \%$, hypertension, diabetes, chronic obstructive pulmonary disease, obstructive sleep apnea, and obesity was seen in the Device Clinic for a routine surveillance of his single-chamber St. Jude Medical (Sylmar, California) Fortify Assura ${ }^{\mathrm{TM}}$ implantable cardioverter defibrillator (ICD) implanted for primary prevention of sudden cardiac death.

This was his second generator having been implanted in May 2014 as a replacement for an earlier generation ICD that had reached its elective replace-

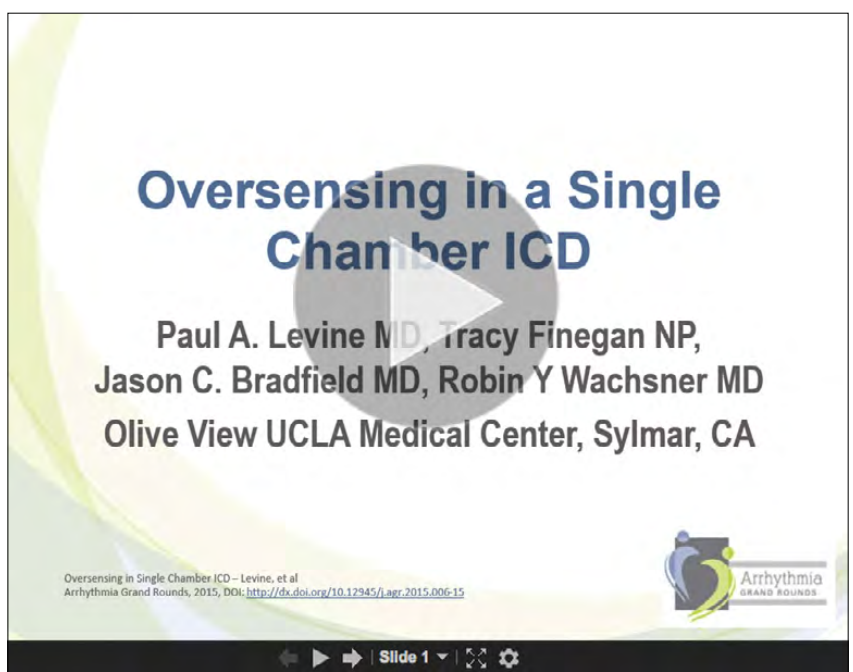

Slideshow. Click on the presentation above to open a new browser window to view the slideshow. Direct link to presentation: http://tiny.cc/agr-0006-15.

ment interval, in part due to high outputs associated with elevated right ventricular (RV) capture thresholds. As such, the RV lead was also replaced at the time of generator replacement. Extensive mapping was required to find a site that provided reasonable capture and sensing thresholds with minimal ectopy. The final position was high on the interventricular septum. In keeping with current practice at many (c) 2015 Arrhythmia Grand Rounds

Published by Science International Corp. ISSN 2326-4012

Accessible online at: http://arrhythmiagr.com/
* Corresponding Author:

Paul A. Levine, MD

25876 The Old Road, PMB 14, Stevenson Ranch, California 91381, USA

Tel. +1 661565 5589, Fax: +1 661254 4702, E-Mail: paul91321@gmail.com 


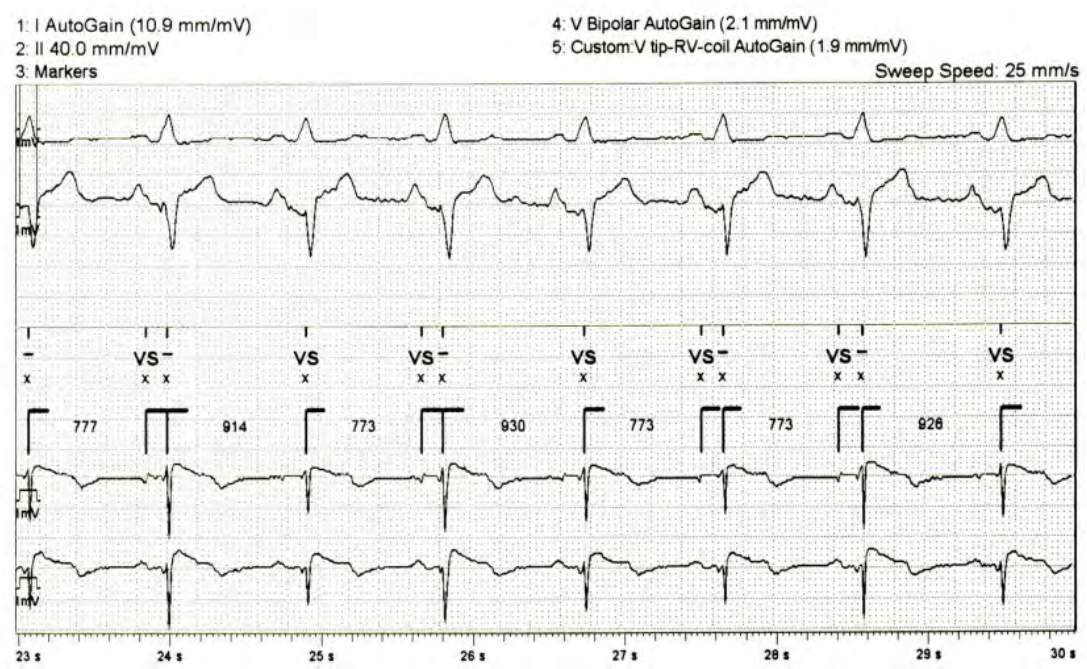

Figure 1. This is episode 59 of the most recent 59 episodes that had been captured by the stored electrogram. All the episodes were reviewed and were similar.

institutions, defibrillation threshold (DFT) testing was not performed, given the primary prevention indication for implant.

Since his prior evaluation 6 months earlier, he had not experienced any syncopal or presyncopal episodes or palpitations. The internal diagnostics of the system reported frequent episodes of "non-sustained R wave oversensing" (Figure 1).

What is the cause of episodes of non-sustained ventricular oversensing (NSO) in this patient? What are the potential consequences of this specific form of oversensing? Do these have to be specifically managed and if so, what might be the options?

\section{Discussion}

There are multiple examples of double counting shown in Figure 1 but, unlike the usual double counting in a single chamber ICD, which is either double counting of a wide native QRS or counting the QRS followed by the T wave, one of the markers clearly aligns with the QRS complex but the other marker

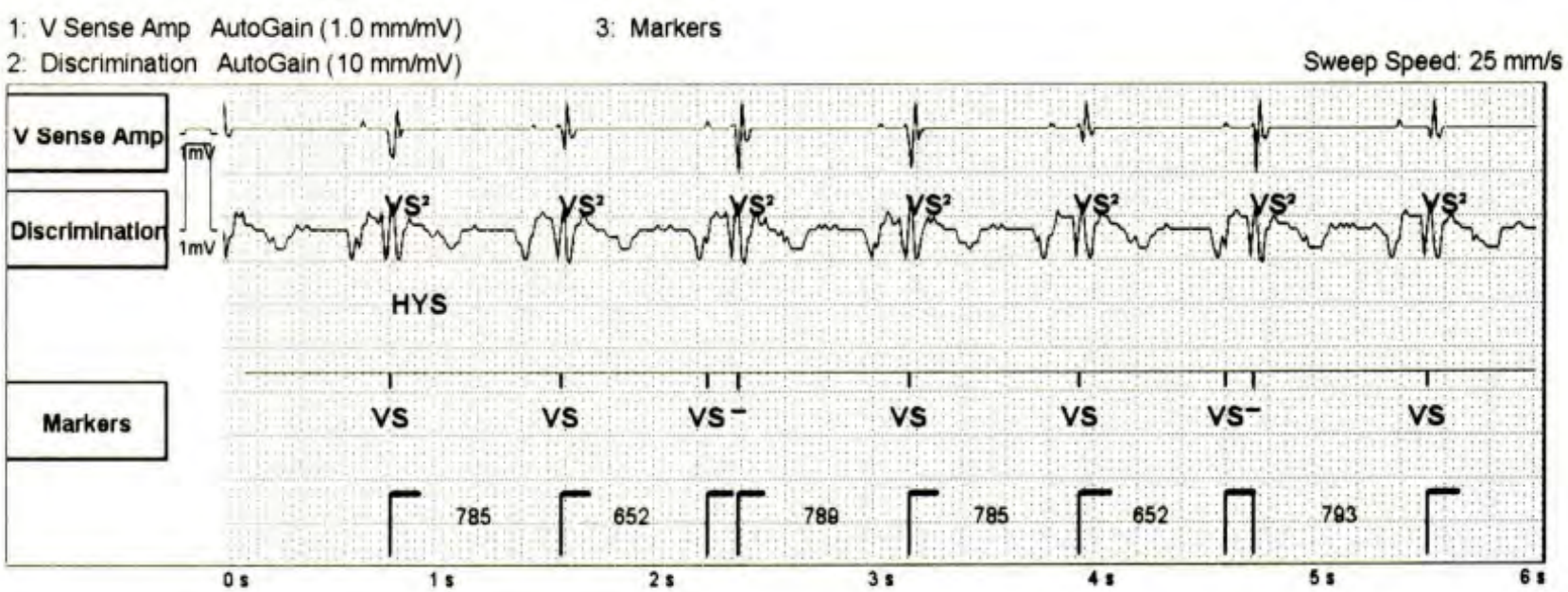

Figure 2. Frozen image recorded at the follow-up session. 


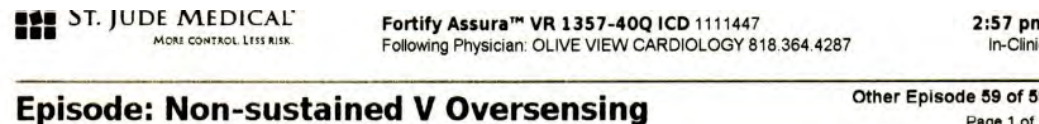

Episode: Non-sustained V Oversensing $\quad$ Other Episode 59 of 59

Jan 7, $20151: 45 \mathrm{pm} \quad \mathrm{VVI}$
Mode
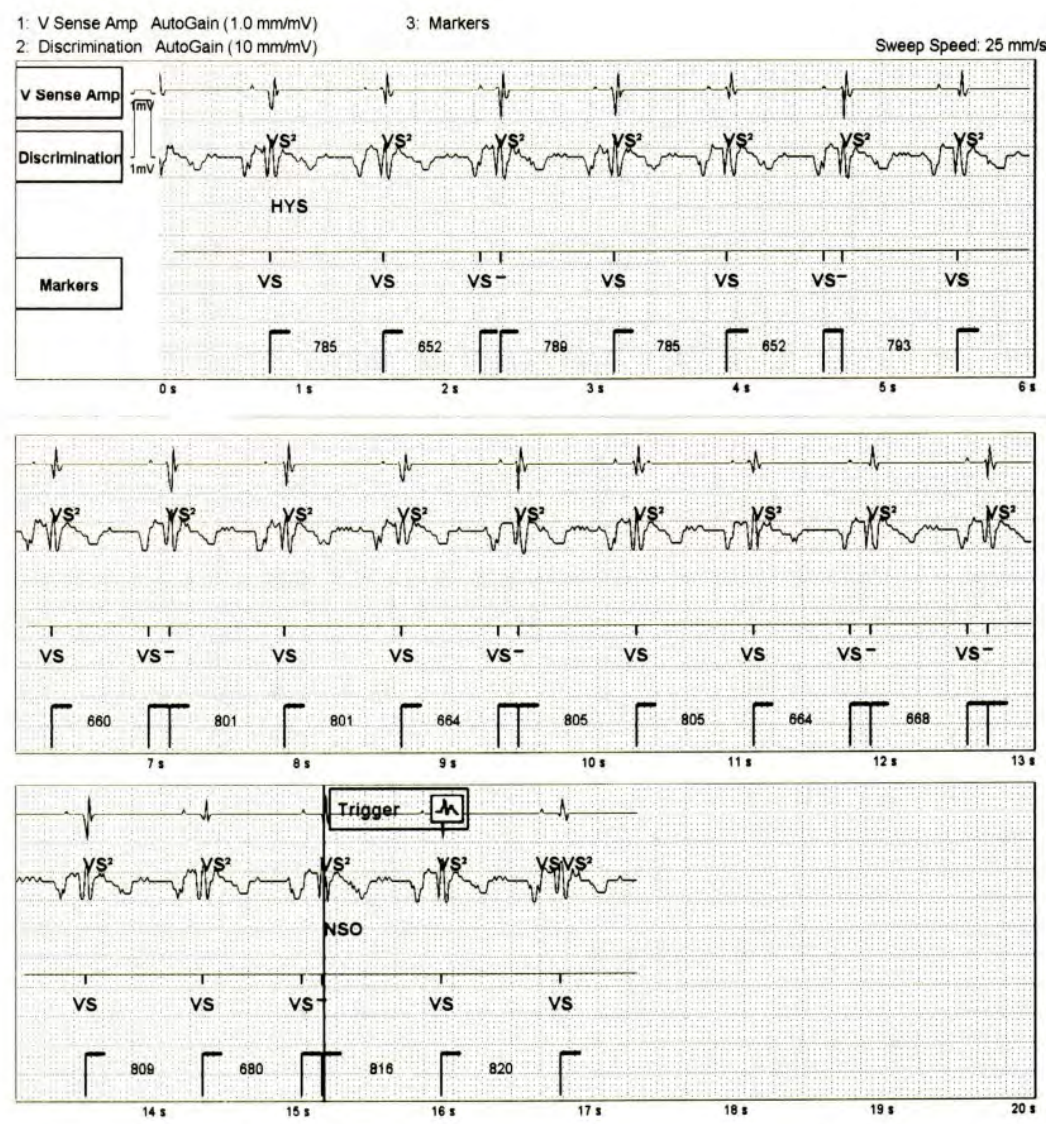

Fortify Assura TM VR $1357-40 \mathrm{~L}$ ICD (1111447 pr 15.02.10) Merlin@ PCS (\#12055470 3330 v18.1_rev_1)

Other Episode 59 of 59 Page 1 of

Figure 3. Stored EGMs demonstrating "V oversensing."The "discriminator" lead configuration is the electrogram recorded between the RV coil and the housing of the ICD. This figure is taken from the first section of Figure 1.

precedes the QRS complex and aligns with the atrial depolarization ( $P$ wave). This was always the case when there were two closely coupled markers, indicating that it was the $P$ wave that was being sensed. Since this was the first signal sensed and was a single chamber ICD, it was labeled VS for ventricular sense. The second complex coincided with the resultant ventricular refractory period; hence, there was no specific label but the small tick mark indicated that it was detected by the system and could be used for the identification of a tachyarrhythmia.

Figure 2 is a frozen image recorded at the follow-up session. As such, in addition to the marker channel notations, there are two surface lead ECGs (I and II), a ventricular bipolar electrogram and a custom $V$ tip to RV coil electrogram. Note that the first VS marker actually aligns with the $P$ wave. In the bipolar ventricular electrogram ( $4^{\text {th }}$ channel), there is a small deflection that is seen preceding the ventricular electrogram and the physically larger of these signals both align 
with the $P$ wave on the surface ECG as well as with the initial ventricular sense marker. Something similar is seen on the stored EGMs captured to document the NSO episodes.

\section{Clinical Significance of Far Field P Wave (FFPW) Oversensing}

In a standard pacemaker, if the $P$ wave is sensed on the ventricular channel, it will inhibit the ventricular output and recycle the timing cycle. If the indication for pacing was sinus node dysfunction with intact atrio-ventricular (AV) nodal conduction, the patient is likely to be asymptomatic. If the indication for pacing was high grade AV block, the patient may be highly symptomatic inhibition of the ventricular channel [1-3] resulting in the loss of ventricular pacing similar to crosstalk first reported in the generation of dual-bipolar DVI pacemakers that did not have a ventricular blanking period triggered by the atrial output pulse [4].

In an ICD, as in this case (Figure 3 ), if the FFPW oversensing was more consistent, this could be interpreted as VT or VF triggering inappropriate high voltage therapy as has occurred with $T$ wave oversensing [5-7]. While this has not yet occurred, it is a potential concern given the frequency with which it seems to be occurring.

\section{How Might FFPW Sensing Be Managed?}

There are a number of options. The relative size of the signal on the telemetered EGM can be misleading, as this is easily influenced by the gain of the EGM independent of what the device is actually seeing. There seems to be a qualitative relationship in that the physically larger EGMs are the ones that correlate with the VS markers. One cannot easily measure the amplitude of the FFPW signal unless it was occurring sufficiently frequently and then one could manually reduce the sensitivity while observing the rhythm. If the episodes disappear at a given programmed sensitivity, the signal amplitude is just below that last value.

One can elect to simply monitor the patient if the observation has not yet triggered any inappropriate therapy. However, if FFPW oversensing has triggered a diagnosis of VT or VF resulting in inappropriate therapy delivery, then more aggressive intervention is required. ICDs have relatively little programmability with respect to sensitivity settings on the high voltage channel. Even if the sinus $R$ wave was greater than $20 \mathrm{mV}$, the least sensitive setting for most ICDs is in the range of 1.0 to $1.3 \mathrm{mV}$ in that the signal amplitude associated with fibrillation can be very small.

Assuming that at a programmed value of $1.0 \mathrm{mV}$, there is apparent resolution of FFPW oversensing, but it is still intermittently, present at $0.8 \mathrm{mV}$. This may be misleading in that the orientation of the lead with respect to the generated signals inside the heart may change with changing body positions or if the atrial focus shifts as may occur with sinus tachycardia or the development of an ectopic atrial rhythm. So measurements made at rest and in a supine position may not reflect signals occurring in other physiologic states. If one wants to reduce the ventricular sensitivity in an effort to eliminate the FFPW oversensing, it will be necessary to perform DFT testing.

When DFT testing is performed in most labs, the ventricular sensitivity is programmed to the least sensitive value, VF is induced and the system observed with respect to detection and termination of VF. If the system does not consistently recognize the VF signals, the patient can be rescued using the external defibrillator. If this is done at implant with failed detection of VF, one could potential reposition the lead in an effort to locate a better position. In this patient, finding a stable lead position with good electrical parameters was a challenge. If one knew that the induced VF was successfully detected at the least sensitive programmed sensitivity, one could then program to this value if it was effective in reducing, if not eliminating, the FFPW oversensing at the time that it was first observed. The operator could then be reasonably confident that this intervention will not compromise VF detection. However, there are some caveats with respect to DFT testing. "Induced VF" during DFT testing is not necessarily the equivalent to clinical VF and, therefore, while induction of sensed VF at the lowest sensitivity during DFT testing provides increased security for successful defibrillation, it does not guarantee success clinically as numerous factors such as acute heart failure, electrolyte abnormalities, and other factors can contribute to clinical VF that may not be present at the time of induced VF. 
If DFT testing was never performed and FFPW oversensing caused the misdiagnosis of VT/VF with therapy delivery, the patient will need to be admitted for DFT testing to know if it is safe to program to a less sensitive setting. If reducing the sensitivity as much as allowed by the pulse generator does not eliminate the FFPW oversensing and had resulted in inappropriate therapy, then an operative procedure would be necessary to reposition the lead or since a chronic lead may be fibrosed in place and manipulation of the lead may cause physical damage to the lead.

If the lead was one of the new in-line DF4 leads, the entire lead would need to be repositioned or replaced. If the lead was a standard ICD lead with two or three ter- minal pins, a standard pace/sense lead could be placed if necessary while leaving the high voltage lead in its current position rather than having to try to extract it with its attendant morbidity and potential physical damage to the lead precluding its being repositioned.

\section{Conflict of Interest}

The authors have no conflict of interest relevant to this publication.

\section{Comment on this Article or Ask a Question}

\section{References}

1. Van Gelder BM, Bracke FALE, el Gamal MIH. $P$ wave oversensing in a unipolar VVI pacemaker. PACE. 1995;18:370-374. DOI: 10.1111/j.1540-8159.1995.tb02529.x

2. Levine PA. Clinical manifestations of lead insulation defects. J Electrophysiol. 1987;1:144-155. DOI: 10.1046/j.14609592.2002.00504.x

3. Garrigue S, Barold SS, Clementy J. Unexpected ventricular asystole in a DDD pacemaker: What is the mechanism? PACE. 2002;25:504-506. DOI: 10.1378/ chest.63.5.783

4. Furman $S$, Reicher-Reiss $H$, Escher DJW. Atrio-ventricular sequential pacing and pacemakers. Chest. 1973;63:783-786. DOI: 10.1023/A:1014132408411
5. Barold SS, Garrigue S, Clementy J. Farfield $P$ wave sensing by the right ventricular lead of conventional dual chamber pacemakers. J Interven Card Electrophysiol. 2002;6:77-80. DOI: 10.1111/j.15408159.2012.03502.x

6. Barold SS, Kucher A, van Heuverswyn F. Timmers L, Stroobandt RX, Unusual cause of far-field atrial sensing by the ventricular lead of a dual chamber defibrillator: What is the mechanism? PACE. 2013;36:501-504. DOI: $10.1111 /$ j.1540-8159.2012.03502.x

7. Vollman D, Luthje L, Zabel M. Far-field oversensing of atrial signals: an unusual cause for very short $\mathrm{V}-\mathrm{V}$ intervals and inappropriate implantable cardioverter defibrillator therapy. Europace. 2013;10:1009-1011. DOI: 10.1093/Europace/eun103.
Cite this article as: Levine PA, Finegan T, Bradfield JC, Wachsner RY. Oversensing in a Single Chamber Implantable Cardioverter Defibrillator. Arrhythmia Grand Rounds 2015;1(2):67-71. DOI: $\quad$ http://dx.doi.org/10.12945/j. agr.2015.006-15

\section{Addendum}

Since the submission of this manuscript, the patient returned with a deterioration in capture and sensing thresholds and an increase in the frequency of non-sustained over-sensing but without inappropriate episodes of VT or VF as well as no episodes of high voltage therapy. A follow-up chest x-ray demonstrated lead displacement with the RV coil now located in the right atrium. The radiography film was underpenetrated and would not present well in publication. In view of this, the patient is scheduled for admission to explant the RV high voltage lead and replace it with a new lead. 